\title{
Prácticas pedagógicas apoyadas por tecnologías móviles: oportunidades para potenciar el aprendizaje de la población estudiantil
}

\author{
Pedagogical practices supported by mobile technologies: \\ opportunities to enhance the learning of the student population
}

\author{
Melissa Villalobos-García \\ Investigadora, Unidad de Evaluación \\ Fundación Omar Dengo \\ San José, Costa Rica \\ melissa.villalobos@fod.ac.cr \\ Olmer Núñéz-Sosa \\ Investigador, Unidad de Evaluación \\ Fundación Omar Dengo \\ San José, Costa Rica \\ olmer.nunez@fod.ac.cr
}

Recibido - Received: 14 / 02 / 2020

Corregido - Revised: 05 / 05 / 2020

Aceptado - Accepted: 01 / 06 / 2020

\section{DOI: https://doi.org/10.22458/ie.v22i32.2806 \\ URL: https://revistas.uned.ac.cr/index.php/innovaciones}

\begin{abstract}
Resumen: Con el fin de identificar aspectos clave para potenciar el aprovechamiento de las tecnologías móviles en los procesos de aprendizaje, se plantea un estudio de alcance descriptivo en el que se comparan condiciones socioeconómicas, educativas y de aprovechamiento de estos recursos en dos poblaciones de estudiantes de secundaria: participantes con al menos dos años de experiencia en el uso de tecnologías móviles en clase y participantes que no contaban con dicha experiencia. Desde un enfoque comparativo entre las poblaciones, se identificó que las personas estudiantes que reciben apoyo pedagógico para incorporar tecnologías móviles en sus procesos de aprendizaje tienden a considerarse usuarios más competentes de dichos recursos; muestran mayor repertorio de conocimiento sobre herramientas útiles en el ámbito educativo, y evidencian mejores condiciones para usar de manera segura espacios de ocio como las redes sociales. Se concluye que una de las mayores oportunidades para potenciar el aprovechamiento de las tecnologías móviles en los procesos de aprendizaje se relaciona con el rol que tienen sus docentes en ello, por lo cual favorecer el desarrollo de apropiación tecnológica en la población docente resulta clave dentro de este tipo de propuestas educativas.
\end{abstract}

Palabras clave:Tecnología educacional; Enseñanza asistida por ordenador; Estudiante de secundaria; Educación sobre medios de comunicación; Educación y ocio; Investigación pedagógica; Proceso de aprendizaje

Summary: In order to identify key aspects to enhance the use of mobile technologies in learning processes, a descriptive study is proposed comparing socioeconomic, educational and good use conditions of these resources in two populations of high school students: participants with at least two years of experience in using mobile technologies in class and participants who did not have such experience. From a comparative approach between the populations, it was identified that the students who receive pedagogical support to incorporate mobile technologies in their learning processes tend to consider themselves more competent users of said resources. They show a greater repertoire of knowledge about useful tools in the educational field, and show better conditions to safely use leisure spaces such as social networks. It is concluded that one of the greatest opportunities to enhance the use of mobile technologies in learning processes is related to the role that their teachers have in it, therefore favoring the development of technological appropriation in the teaching population is key within this type of educational proposals.

Keywords: Educational technology; Computer aided instruction; High-school student; Media education; Education and leisure; Pedagogical research; Learning process 


\section{INTRODUCCIÓN}

Crompton (2013) define el aprendizaje apoyado con tecnologías móviles como un aprendizaje a través de contextos múltiples, que se da por medio de interacciones sociales y de contenido y en el que se hace uso de dispositivos electrónicos personales. Desde el año 2011, el Programa Nacional de Informática Educativa del Ministerio de Educación Pública y la Fundación Omar Dengo (PRONIE MEP-FOD) ha trabajado en la definición e implementación de un modelo educativo orientado al Aprendizaje con Tecnologías Móviles (ATM).

Desde un enfoque de equidad, el modelo educativo de ATM ha adquirido protagonismo dentro del Programa, privilegiando "zonas de la ruralidad dispersa, como una estrategia de mitigación de la brecha digital que sufren los habitantes de esas regiones" (FOD, 2016a, p. 94). Sin embargo, más allá del equipamiento, el modelo se caracteriza por brindar apoyo pedagógico y formación docente para que la población estudiantil pueda aprovechar las tecnologías móviles para favorecer sus aprendizajes.

La incorporación de tecnología en los procesos de aprendizaje debe darse desde una perspectiva en la que se visualice "como herramienta de construcción del conocimiento para que los alumnos aprendan con ellas y no sólo de ellas" (Said et ál., 2015, p. 30). Partiendo de ello, el enfoque pedagógico del modelo de ATM busca que los y las estudiantes desarrollen apropiación tecnológica y utilicen la tecnología para comprender y crear conocimiento mientras aprenden colaborativamente y controlan su propio proceso de aprendizaje (FOD, 2016a). Sobre el concepto de apropiación tecnológica, desde el marco de resultados de aprendizaje esperados en las personas estudiantes del PRONIE MEP-FOD, se define:

Apropiarse de las tecnologías digitales implica, entonces, no sólo tener algún contacto con ellas, o saber usarlas, sino también conocer de ellas lo necesario y suficiente para lograr sacar ventaja de sus potencialidades en las diversas situaciones que se nos presentan en la vida cotidiana. (Fundación Omar Dengo, 2006, p. 18)

La apropiación se presenta a nivel individual, pero también es un fenómeno social "por el esfuerzo que hace la sociedad, como un todo, para hacerla posible" (FOD, 2016a, p. 82). Así, la apropiación se ve favorecida por una serie de experiencias que median el proceso de conocer y saber sacar ventaja de las tecnologías digitales para distintos fines propuestos dentro de la práctica educativa. Siguiendo a Gómez (2008), "la práctica educativa es una actividad compleja que está determinada por una multiplicidad de factores, entre ellos: las características de la institución, las experiencias previas de los alumnos y profesores, así como la capacitación que han recibido estos últimos" (p. 30). De esta manera, conocer qué factores intervienen en el entorno de la población estudiantil resulta clave para contar con propuestas educativas coherentes con el entorno.

Said et ál. (2015) señalan la necesidad de contar con ciertas "condiciones escolares para integrar las TIC en las instituciones educativas con el propósito de obtener impactos significativos en el aprendizaje de los estudiantes" (p. 13). Para ello, el Programa ha trabajado en identificar y caracterizar aspectos clave para el éxito de sus propuestas educativas, dentro de los que destacan el desarrollo de estudiantes más autónomos y activos, el trabajo dentro de una cultura de aprendizaje e innovación, así como contar con docentes motivados y preparados que estén acompañados por una dirección que lidere y una comunidad que apoye (FOD, 2016a). Si bien, es posible que las condiciones sean variables dependiendo de la localización del centro educativo, hay algunas que son imprescindibles. Al respecto:

Garantizar el acceso adecuado a los recursos TIC como aspecto clave donde prima la existencia de infraestructura física, el tiempo para el uso de los equipos, la conectividad y la calidad de los 
recursos tecnológicos, contribuyen a la creación de ambientes propicios para el uso efectivo de las TIC. (Shelwyn, citado en Said et ál., 2015, p. 13)

Gutiérrez (2007; citado en Said et ál., 2015) afirma que la integración efectiva de las tecnologías digitales en el aprendizaje está condicionada por la forma en la que se utilicen a nivel didáctico, teniendo en cuenta los requerimientos tecnológicos y pedagógicos para obtener de ellas un mayor potencial. Por ello, aunque interesa identificar las condiciones de los y las estudiantes (socioeconómicas, desempeños, actitudes y acceso a TIC), resulta igualmente relevante perfilar las prácticas educativas de las que participan, en términos de las características de sus docentes, las dinámicas de clase, las prácticas pedagógicas y las actitudes y expectativas con respecto al uso de tecnología (Said et ál., 2015).

Partiendo de lo anterior, el presente estudio define como objetivo general: Identificar aspectos clave útiles para potenciar el aprovechamiento de las tecnologías móviles en los procesos de aprendizaje. Con el fin de lograr la meta, se delimitan los siguientes objetivos específicos:

1. Comparar las condiciones socioeconómicas, educativas y de aprovechamiento de las tecnologías móviles en dos poblaciones de estudiantes de secundaria (con y sin experiencia en el uso de estos recursos).

2. Caracterizar los aspectos clave identificados en los casos de mayor aprovechamiento de las tecnologías móviles como una vía para potenciar su incorporación en las prácticas pedagógicas.

\section{MATERIALES Y MÉTODOS}

\section{Diseño}

Se definió un estudio de tipo mixto de triangulación concurrente, el cual fue útil para realizar una validación cruzada entre los datos cuantitativos y los cualitativos recopilados para el estudio, aprovechando las ventajas de cada método (Hernández, Fernández y Baptista, 2010).

En este caso particular, se presentó un proceso de recolección de datos cuantitativos y cualitativos de estudiantes, sus docentes y los y las directoras de los centros educativos participantes del estudio; para lo cual se tomó en cuenta un grupo de participantes de una de las propuestas del modelo educativo de ATM del PRONIE MEP-FOD y un grupo de "no participantes" en esta propuesta.

El alcance del estudio es descriptivo puesto que se definieron variables útiles para identificar las condiciones asociadas al tipo de aprovechamiento de las tecnologías móviles, como base para la identificación de oportunidades para potenciar el aporte de dichos recursos en los procesos de aprendizaje de las personas estudiantes. Desde esta perspectiva, se estudiaron diferentes componentes y conceptos, con el objetivo de describirlos y asociarlos para identificar relaciones que permitieran interpretar los datos (Hernández, Fernández y Baptista, 2014).

\section{Estrategia de recolección de datos}

Se desarrollaron instrumentos de tipo cuestionario para la población estudiantil, sus docentes y los y las directoras de los centros educativos, así como un protocolo de observación de clases. En el caso de los cuestionarios, el tipo de ítem utilizado fue principalmente de selección única y, en menor medida, de tipo abierto. Concretamente, se abordaron las siguientes variables: 
1. Cuestionario para estudiantes: características relativas a condiciones socioeconómicas, acceso a tecnología (entendido como la tenencia de dispositivos tecnológicos y la frecuencia de uso), aprovechamiento de las tecnologías móviles para aprender (desempeños iniciales al incorporar tecnologías móviles en los procesos de aprendizaje) y actitudes y expectativas hacia el uso de la tecnología (disposición de los estudiantes hacia la incorporación de tecnologías móviles en sus actividades educativas y de ocio).

2. Cuestionario para docentes: perfil profesional (datos relacionados con formación y experiencia profesional), acceso a recursos tecnológicos (entendido como la tenencia de dispositivos tecnológicos y la frecuencia de uso), actitudes y expectativas hacia el uso la tecnología (disposición hacia la incorporación de tecnologías móviles en las actividades educativas) y prácticas pedagógicas (actividades que desarrollan para favorecer procesos de aprendizaje con o sin uso de tecnologías móviles).

3. Cuestionario para directores: caracterización de las comunidades (factores socioeconómicos de las comunidades en las que se encuentran los centros educativos); características de liderazgo educativo (tipo de apoyo que brindan a sus docentes en los procesos educativos) y actitudes y expectativas hacia el uso de la tecnología (disposición hacia la incorporación de tecnologías móviles en las actividades educativas).

4. Protocolo de observación de clases: incluía espacios para registro de información, principalmente cualitativa relacionada con el aprovechamiento de las tecnologías móviles en las prácticas pedagógicas y en las actividades de aprendizaje.

Todos los instrumentos fueron sometidos a procesos de pilotaje con el fin de valorar aspectos de coherencia interna, así como de coherencia entre los instrumentos y el objetivo del estudio. Los datos fueron recolectados durante los meses de abril y julio del año 2018, en un proceso a cargo del equipo de investigación de la FOD.

\section{Muestra}

La población del estudio contempló a estudiantes de octavo grado de la Educación General Básica pertenecientes a centros educativos beneficiados por el PRONIE MEP-FOD dentro de una de las propuestas educativas del Área de Aprendizaje con Tecnologías Móviles. El diseño muestral fue de tipo no probabilístico intencionado, con dos categorías para la selección de los centros educativos:

- Grupo A: centros educativos que iniciaron la implementación de la propuesta educativa de ATM en el año 2014.

- Grupo B: centros educativos en condición de seleccionados para la instalación, es decir, que aún no habían sido beneficiados por la propuesta educativa, pero estaban a la espera de iniciar.

Se seleccionaron tres centros educativos del Grupo B y se les asignó una institución del Grupo A que funcionara como par para realizar la comparación. La elección de los pares se realizó a partir de criterios como la localización, la zona, el tipo de centro educativo y la cantidad de matrícula en sétimo grado en el 2017 (como proyección del aproximado de estudiantes en octavo en 2018). En total se contó con un total de seis centros educativos, cuyas características se muestran en la tabla 1: 
TABLA 1

Ubicación y características de los centros educativos participantes en el estudio

\begin{tabular}{ccccccc} 
Colegio & Grupo & Cantón & Distrito & Zona & $\begin{array}{c}\text { Matrícula } \mathbf{7}^{\circ} \\
\text { en 2017 }\end{array}$ & $\begin{array}{c}\text { IDS* } \\
\text { Cantonal }\end{array}$ \\
\hline 1 & A & Grecia & Grecia & Urbano & 85 & 74,4 \\
2 & B & Limón & Limón & Urbano & 107 & 26,1 \\
3 & A & Liberia & Cañas Dulces & Rural & 61 & 43,4 \\
4 & B & Puntarenas & Lepanto & Rural & 35 & 40,5 \\
5 & A & Acosta & Sabanillas & Rural & 54 & 45,1 \\
6 & B & Sarapiquí & Horquetas & Rural & 47 & 0,7 \\
\hline
\end{tabular}

*IDS: Índice de Desarrollo Social Cantonal.

Fuente: Ministerio de Planificación Nacional y Política Económica [MIDEPLAN], (2018).

En cada centro educativo se aplicó un cuestionario a la población estudiantil de octavo grado, a sus docentes de Español y Matemática, así como a los y las directoras. Además, se realizó una observación de clase por cada una de las materias citadas, en octavo grado. En total, se contó con una muestra de 250 estudiantes ( 150 del Grupo A y 100 del Grupo B), 6 directores y 11 docentes, así como un total de 10 observaciones efectivas (en dos casos no se logró observar la clase de Español).

\section{Análisis de la información}

El análisis de la información se realizó desde un enfoque estadístico descriptivo, que permitió analizar la información de los cuestionarios para describir las condiciones de las personas estudiantes, sus docentes y los y las directoras, en las dimensiones y variables analizadas.

En paralelo, se realizó un análisis de información bajo la metodología cualitativa que implicó la categorización interpretativa, posterior a la sistematización de la información recopilada por medio de las observaciones de clase y las preguntas de tipo abierto. Toda la información sistematizada de las diferentes fuentes y técnicas aplicadas fue sometida a un proceso de triangulación para caracterizar a la población y compararla, con el fin de buscar aspectos clave sobre el aprovechamiento de las tecnologías móviles, en ambos grupos. Cabe mencionar que, debido a que se trató de una muestra no probabilística, los resultados no son generalizables para toda la población beneficiaria del modelo educativo de ATM del PRONIE MEP-FOD.

\section{RESULTADOS}

\section{Caracterización de los participantes}

En la tabla 2 se muestra una síntesis de la caracterización de las comunidades y los y las participantes del estudio. 
TABLA 2

Perfil de los y las participantes

\begin{tabular}{|c|c|}
\hline :ipantes & Características \\
\hline Comunidades & $\begin{array}{l}\text { - } \mathrm{n}=6 \text { (2 urbanos y } 4 \text { rurales). } \\
\text { - Localizadas principalmente en cantones con bajo índice de desarrollo social. } \\
\text { - Las principales ocupaciones de sus habitantes se clasifican en la categoría de agricultores y trabajadores calificados } \\
\text { agropecuarios, forestales y pesqueros, según el Instituto Nacional de Estadística y Censo (INEC, 2014). }\end{array}$ \\
\hline Directores & $\begin{array}{l}\text { - } \mathrm{n}=6 \text { (5 hombres y } 1 \text { mujer) con } \bar{x} \text { de edad = 48,83 años. } \\
\text { - } \text { Tienen al menos } 12 \text { años de experiencia profesional. } \\
\text { - } \quad \text { La mayoen estudía cuenta con computadora en su casa ( } 5 \text { de } 6 \text { ) y se consideran usuarios intermedios de la tecnología. } \\
\text { - Tienen poca participación en ofertas de desarrollo profesional sobre uso de tecnología (sólo } 2 \text { de } 6 \text { reportaron } \\
\text { haber sido capacitados). } \\
\text { - Muestran disposición para favorecer la incorporación de tecnologías móviles en los procesos educativos. }\end{array}$ \\
\hline Docentes & $\begin{array}{l}\text { - } \mathrm{n}=11 \text { (7 mujeres y } 4 \text { hombres) con } \bar{x} \text { de edad = 36,54 años. } \\
\text { - Tienen más de siete años de experiencia como docentes y son de Matemática (6 de 11) y Español (5 de 11). } \\
\text { - Lgresados principalmente de universidades privadas (10 de 11), de licenciatura (4 de 11) y maestría (4 de 11). } \\
\text { usuarios intermedios-avanzados de la tecnología. } \\
\text { - Los del Grupo A cuentan con capacitación para utilizar tecnología en los procesos de aprendizaje (como parte de } \\
\text { su participación en la propuesta del PRONIE MEP-FOD). Mientras que los del Grupo B se encontraban a la espera de } \\
\text { la capacitación. } \\
\text { - Los del Grupo A reportaron con mayor frecuencia que su director favorece su desarrollo profesional (5 de } 5 \text { docen- } \\
\text { tes del grupo A y } 3 \text { de } 6 \text { del grupo B) y que los motiva a aprovechar los recursos educativos del colegio (5 de } 5 \text { del } \\
\text { grupo A y } 3 \text { de } 6 \text { del Grupo B). }\end{array}$ \\
\hline Estudiantes & $\begin{array}{l}\left.\text { - } \mathrm{n}=250 \text { ( } \mathrm{n}_{\text {Grupo A }}: 150, \mathrm{n}_{\text {Grupo }}: 100\right) \\
\text { - } 53 \% \text { hombres y } 47 \% \text { mujeres con } \bar{x} \text { de edad = } 14,28 \text { años. } \\
\text { - No presentan condiciones de hacinamiento en el hogar** (sólo el } 3 \% \text { reportó esta condición). } \\
\text { - Tienen importante acceso a recursos tecnológicos como: celular }(99 \%) \text {, televisión por cable (87\%) y computadora } \\
\text { (75\%). } \\
\text { - El } 76 \% \text { reportó acceder a Internet todos los días desde algún dispositivo tecnológico. }\end{array}$ \\
\hline
\end{tabular}

*Se define como límite inferior o nivel muy bajo desarrollo relativo el rango de 0,0. a 45,5 en el IDS para el 2017 (MIDEPLAN, 2018 ).

**El índice de hacinamiento corresponde a la división del número de personas que habitan en el hogar entre el número de habitaciones para dormir.

Fuente: Elaboración propia a partir de los hallazgos del estudio (2019).

Tanto los y las directoras como la población docente muestran buenas actitudes hacia el uso de las tecnologías digitales en la educación. Coinciden en que se debe hacer lo posible para integrar los recursos en las clases ( 5 de 6 directores y 9 de 11 docentes) y que el estudiantado aprenda a utilizarlos es tan importante como que aprenda otros temas (5 de 6 directores y 9 de 11 docentes). Sobre los objetivos de incorporar la tecnología en la educación, los y las directoras mostraron una visión más amplia al coincidir en que favorece que las personas estudiantes desarrollen habilidades necesarias para la sociedad actual (5 de 6). Mientras que la población de docentes lo enfoca más en lo inmediato, al valorar que es útil para que la población estudiantil aprenda mejor en clase con actividades innovadoras y atractivas (9 de 11). Estas actitudes positivas de los y las directores y la población docente coinciden con las de la población estudiantil, quienes tienden a valorar que la tecnología constituye un apoyo importante para sus procesos de aprendizaje (ver figura 1). 
Figura 1. Percepción de la población estudiantil sobre el aporte del uso de computadoras a sus aprendizajes ¿Qué tanto me aporta contar con computadoras para aprender?

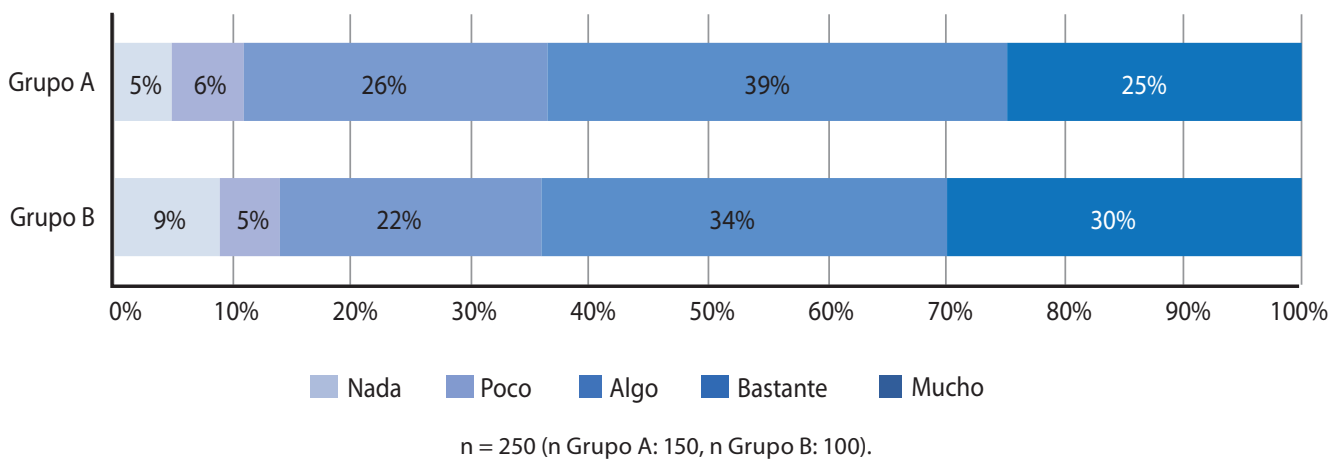

Fuente: Elaboración propia a partir de los hallazgos del estudio (2019).

\section{Prácticas pedagógicas en el centro educativo}

Se observó que las personas estudiantes del Grupo B mostraron una mayor tendencia a trabajar individualmente, mientras que los del Grupo A trabajaban más en colaboración, lo cual concuerda con uno de los objetivos del modelo educativo de ATM, orientado a la promoción del trabajo colaborativo.

Dentro de las actividades que la población estudiantil reportó como más frecuentes se encontró: copiar la materia que el docente facilita en la pizarra o por dictado (reportado por más de $85 \%$ de la población estudiantil de ambos grupos), realizar prácticas con libros o material impreso (más de $80 \%$ de ambos grupos) y trabajar individualmente en redacciones, lecturas o mapas conceptuales (más de $50 \%$ de ambos grupos). El uso de las tecnologías móviles no destacó dentro de las actividades más frecuentemente reportadas por esta población estudiantil.

Con respecto a las estrategias pedagógicas, a partir de las observaciones de clase realizadas, se encontró que ambos grupos de docentes tienden a utilizar estrategias de mediación más directivas y tradicionales como la promoción del trabajo individual (en 8 de 10 observaciones de clase) y las explicaciones magistrales (6 de 10 observaciones de clase). Aunque el estudiantado reportó poca frecuencia de uso de recursos tecnológicos en las clases, la población docente del Grupo A sí reportó utilizar la computadora y el celular para desarrollar actividades educativas como: solicitar búsquedas de información ( 5 de 5 docentes) y crear resúmenes para favorecer el aprendizaje de la población estudiantil ( 5 de 5 docentes). Por su parte, los docentes del Grupo B reportaron no contar con las condiciones necesarias para realizar tal uso con sus estudiantes pues carecían todavía de las tecnologías móviles de la propuesta del PRONIE MEP-FOD en su centro educativo.

Un hallazgo común dentro del grupo de docentes, es que reportaron apoyar su gestión de los procesos educativos con herramientas como: Word (10 de 11 docentes), YouTube (8 de 11) y PowerPoint (7 de 11), desde una perspectiva más administrativa, dentro de la que destacaron la elaboración de documentos de control (como registros o rúbricas) y el hacer pruebas escritas y planeamientos didácticos (en promedio 8 de 11 docentes reporta hacer siempre estas actividades). Por otra parte, señalaron que no suelen apoyarse en la tecnología para favorecer la comunicación y colaboración con sus colegas o participar en comunidades de aprendizaje en línea (7 de 11 docentes reportan nunca realizar dichas actividades). Vinculado con esto, ambos grupos de docentes consideran que para hacer un buen aprovechamiento de las tecnologías móviles es necesario contar con adecuado acceso a Internet (6 de 11 docentes) y con capacitación adecuada para ello (3 de 6 docentes del Grupo B, es decir, que esto fue mencionado por docentes que aún no habían sido capacitados como parte de su ingreso a la propuesta educativa del Programa). 


\section{Aprovechamiento de las tecnologías móviles para aprender}

En la frecuencia de uso de la computadora por parte de la población estudiantil, tanto en su tiempo libre como en actividades relacionadas con el estudio, no se encontraron diferencias estadísticamente significativas entre ambos grupos, siendo la tendencia frecuente la siguiente: sobre $85 \%$ reporta utilizar la computadora más de cinco días por semana). Como se muestra en la figura 2, la mayoría de los estudiantes se considera usuario intermedio del recurso, aunque se encontró una diferencia estadísticamente significativa entre ambos grupos, siendo que: con mayor frecuencia la población estudiantil del Grupo A, es decir, los que tenían experiencia dentro del Programa, se consideran usuarios intermedios y avanzados del recurso.

Figura 2. Valoración de la población estudiantil sobre su nivel de uso de las computadoras

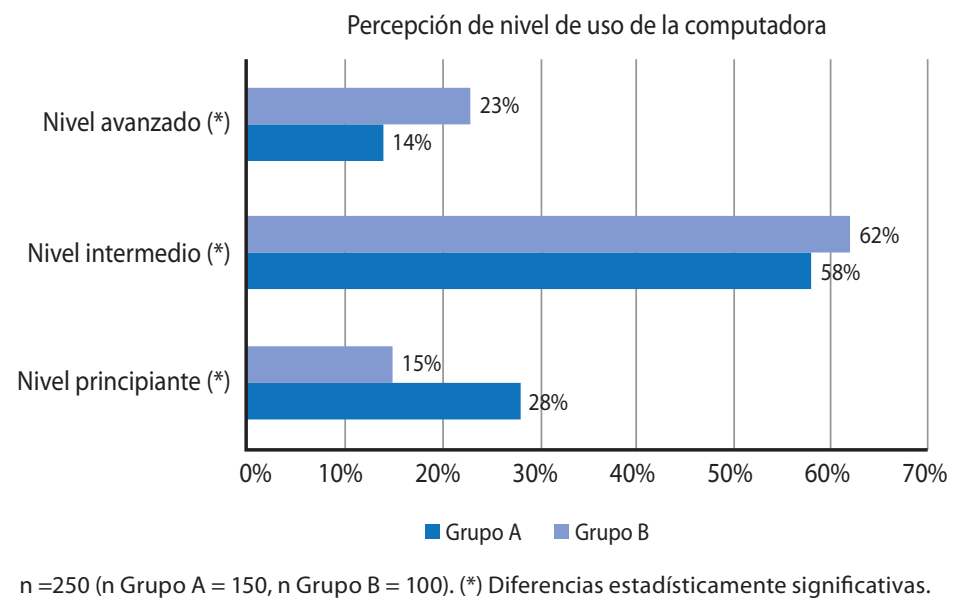

Fuente: Elaboración propia a partir de los hallazgos del estudio (2019).

Así, 97\% del bloque de estudiantes del Grupo A reportó que algún docente de su colegio había utilizado tecnologías móviles en clases, principalmente computadora y celular. En Español, las actividades que reportaron con mayor frecuencia el bloque de estudiantes del Grupo A fueron: desarrollar textos o resumir información (66\% de las personas estudiantes), buscar información en Internet sobre alguna materia (54\%) y proyectar videos (46\%). En Matemáticas, reportaron aprovechar las tecnologías móviles en actividades tales como utilizar herramientas para hacer cálculos (68\%), realizar prácticas con la computadora o celular (41\%) y usar programas específicos de esa materia (38\%). Algunos de estos aspectos se evidenciaron en las observaciones de clases, en las que se advirtió el uso de textos digitales, la realización de búsquedas de información por parte de la población estudiantil, la elaboración de presentaciones con diapositivas, la producción de documentos y la proyección de videos. Mientras que, en el Grupo B, únicamente se observó el uso de la calculadora de los celulares, al no disponer este bloque de estudiantes de las tecnologías móviles de la propuesta educativa del Programa.

Más de 70\% de la población estudiantil que participó en el estudio tiende a relacionar el uso de Internet con aprendizaje educativo y reportan que les sirve para aprender sobre diferentes temas de Matemáticas y Ciencias (reportado por Grupo A), así como vocabulario en Inglés (reportado por Grupo B). Sobre el uso de tecnología para actividades educativas reportaron con mayor frecuencia: enviar mensajes a sus compañeros de clase para discutir asuntos del colegio y buscar información sobre alguna materia (actividades realizadas más de tres días a la semana por alrededor de $70 \%$ de la población estudiantil). Otras actividades como sacar fotografías para un trabajo o redactar un documento fueron reportadas de manera frecuente por cerca de $50 \%$ de participantes de ambos grupos. 
Como se muestra en la figura 3, en algunas herramientas orientadas al ámbito educativo, la población estudiantil del Grupo A considera disponer de un conocimiento mayor que los del Grupo B, siendo esta diferencia estadísticamente significativa.

Figura 3. Percepción de la población estudiantil sobre el propio dominio de herramientas educativas Percepción de dominio intermedio-avanzado

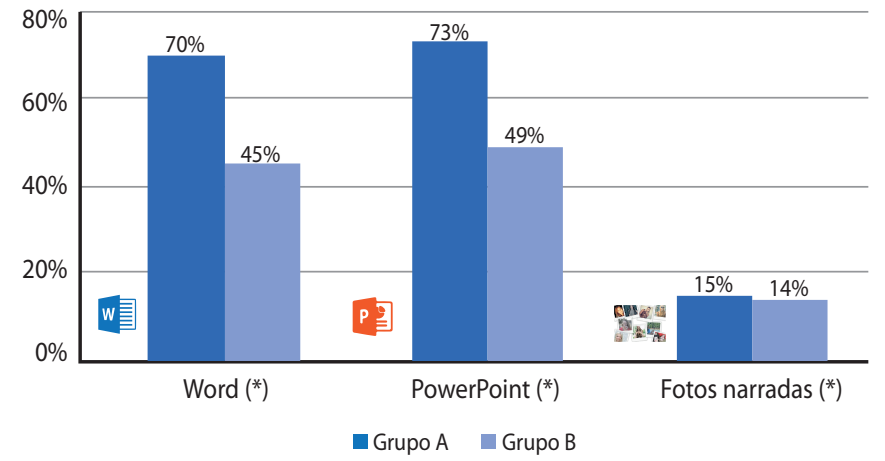

n=250 ( $n$ Grupo $A=150, n$ Grupo B=100). (*) Diferencias estadísticamente significativas

Fuente: Elaboración propia a partir de los hallazgos del estudio (2019).

La población reportó que, en el tiempo libre utilizan las tecnologías móviles para enviar mensajes de texto o chatear con otras personas, revisar redes sociales y escuchar o descargar música más de tres días a la semana (reportado por más de $80 \%$ de los participantes de ambos grupos). Lo anterior, coincide con las herramientas en las que valoran tener un nivel de dominio más avanzado, las cuales se relacionan principalmente con redes sociales, como se muestra en la figura 4.

Figura 4. Percepción de la población estudiantil sobre su propio dominio de herramientas orientadas al ocio

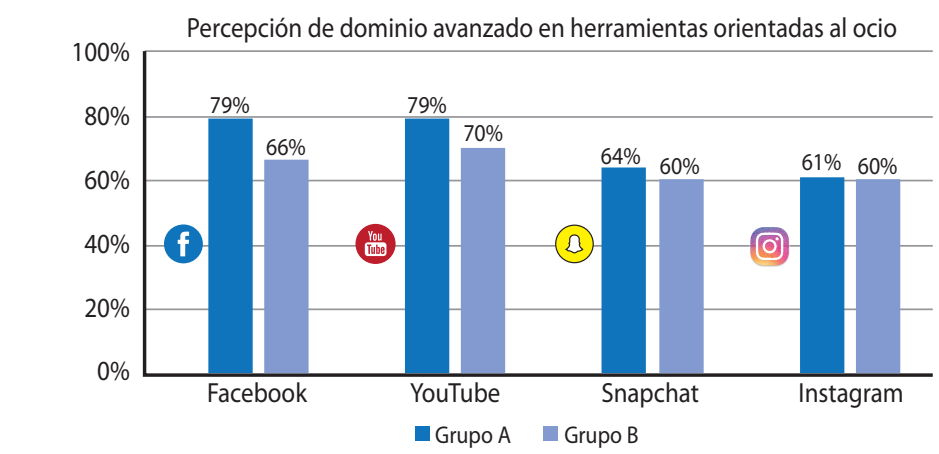

$n=250$ ( $n$ Grupo $A=150, n$ Grupo $B=100$ ). Las diferencias no son estadísticamente significativas.

Fuente: Elaboración propia a partir de los hallazgos del estudio (2019).

Al profundizar sobre los usos de las redes citadas se encuentra que lo principal es compartir fotos o videos ( $60 \%$ del Grupo B y $41 \%$ del Grupo A, siendo esta diferencia estadísticamente significativa). Aunado a que las personas estudiantes del Grupo A reportaron en mayor proporción conocer sobre normas de uso seguro, responsable y efectivo de las tecnologías digitales (38\%, en comparación con apenas 16\% del Grupo B, diferencia estadísticamente significativa), se sugiere que este grupo parece estar en mejores condiciones para hacer un uso más seguro de los espacios. 


\section{CONCLUSIONES}

Al comparar las condiciones socioeconómicas de los participantes del estudio, se evidenció que el perfil socioeconómico bajo es similar en ambos grupos de estudiantes, ante lo cual la participación de estas comunidades en la propuesta educativa del PRONIE MEP-FOD podría considerarse como una contribución al cierre de brechas de acceso a recursos tecnológicos que la población estudiantil de estas zonas podrían mostrar, según el perfil en estudio.

Además, al no encontrar diferencias en el perfil de acceso a otros recursos tecnológicos entre los participantes, un aporte importante que la propuesta educativa de ATM podría realizar es brindar a las personas estudiantes oportunidades para mejorar sus estrategias de aprendizaje mediante el aprovechamiento de los recursos.

Los perfiles evidenciados por las poblaciones estudiantiles y docentes constituyen una fortaleza para el aprovechamiento de las tecnologías móviles en los procesos de aprendizaje. En el caso de los y las directores y la población docente, su experiencia en el trabajo con estudiantes aunado a las actitudes positivas y la autovaloración que hacen de su propio uso de la tecnología podrían ser factores que contribuyan a un mayor aprovechamiento educativo de las tecnologías móviles. Para ello, sería oportuno apoyarlos desde las ofertas de desarrollo profesional con el fin de que cuenten con más y mejores herramientas para alcanzar los objetivos educativos con apoyo de la integración de las tecnologías en su quehacer.

El hallazgo de actitudes positivas de las poblaciones estudiantiles y docentes hacia el uso de tecnologías móviles es consistente con las evidencias de otros estudios realizados con población beneficiaria del Programa (FOD, 2015a; FOD, 2015b; FOD, 2015c y FOD, 2016b). Lo anterior se considera un factor que podría influir positivamente en el aprovechamiento educativo de los recursos (Area, 2005 y Claro, 2010). En esta línea, se encontró una tendencia la población de docentes a valorar de manera positiva el liderazgo del director o directora, lo cual constituye un factor importante, considerando que el rol es fundamental para garantizar un adecuado funcionamiento de los proyectos educativos en cada institución (Ping Lim et ál., 2014).

Por otra parte, al analizar las prácticas pedagógicas, se identificaron estrategias predominantemente tradicionales. El hallazgo sugiere la necesidad de orientar mejor a la población docente sobre posibles caminos para distanciarse de ese tipo de estrategias de mediación y que puedan avanzar hacia las más innovadoras, aprovechando con mayor frecuencia las tecnologías móviles disponibles para apoyar los procesos de aprendizaje.

En este sentido, destaca como un aspecto clave la necesidad de explicitar a la población docente el modelo pedagógico subyacente capaz de generar cambios en los aprendizajes de la población estudiantil (Pérez, 2011).

Otra vía para potenciar el aprovechamiento de las tecnologías móviles en los procesos de aprendizaje podría ser que desde las instituciones se incentive crear y compartir materiales educativos digitales, los cuales puedan ser de utilidad para toda la población docente (Canales y Marqués, 2007).

En el caso de los estudiantes, los hallazgos mostraron que quienes son participantes de la propuesta educativa de ATM evidencian mejores condiciones para utilizar de manera segura las tecnologías móviles en su tiempo libre. Lo anterior se deriva de que estos estudiantes reportaron con mayor frecuencia conocer y aplicar normas de seguridad en espacios como las redes sociales. En la actualidad, el uso de las redes parece tener un importante protagonismo dentro de la cultura digital de la población estudiantil (Del Barrio y Ruiz, 2014) y los participantes del estudio no son la excepción, con lo cual el hallazgo da una 
pista sobre posibles aportes que la propuesta educativa de ATM podría estar brindando al tema de uso seguro de estos espacios.

La población estudiantil del Grupo A mostró una tendencia a autovalorarse con mejor dominio de herramientas tecnológicas útiles dentro del ámbito educativo, en comparación con el Grupo B, que aún no participaban de la propuesta educativa de ATM. Lo anterior evidencia que, aunque la población estudiantil puede tener facilidad para utilizar las tecnologías móviles, en general requieren de apoyo pedagógico para hacer un mayor aprovechamiento de ellas en sus procesos educativos y de aprendizaje.

De igual manera, con respecto al hallazgo de la tendencia al trabajo individual por parte del estudiantado, cabe destacar que la oportunidad de que éstos tengan espacios en los que puedan compartir sus producciones con el grupo se considera clave para empoderarse como líderes de sus procesos de aprendizaje (Canales y Marqués, 2007).

Los hallazgos sobre el tipo de aprovechamiento de las tecnologías móviles que evidenciaron los participantes del estudio, sugieren que una oportunidad clave para potenciar el aprovechamiento está estrechamente relacionado con el rol del docente. Concretamente, en la medida en la que se motive al cuerpo docente a realizar más y mejores usos de las tecnologías móviles se podría favorecer el aprovechamiento por parte de la población estudiantil de los recursos en sus procesos de aprendizaje. Para ello, una orientación constructivista, progresista y centrada en las personas estudiantes, que promueva la creación de proyectos colaborativos de acuerdo con el interés de la población estudiantil, es un aspecto clave para favorecer el aprendizaje (Claro, 2010).

Así, a partir del presente estudio, es posible concluir que la canalización de esfuerzos a favorecer el desarrollo de apropiación tecnológica del cuerpo docente, desde el punto de vista institucional, en el que la apropiación va más allá de saber usar las tecnologías y más bien implica estar en capacidad de sacar ventaja de su potencial en la vida cotidiana (Fundación Omar Dengo, 2006), podría ser uno los mecanismos más efectivos para potenciar el aprovechamiento de estos recursos en los procesos de aprendizaje de las personas estudiantes. 


\section{REFERENCIAS}

Area, M. (2005). Tecnologías de la información y comunicación en el sistema escolar. Una revisión de las líneas de investigación. Relieve, 11(1), 3-25. Recuperado de http://www.uv.es/RELIEVE/v11n1/ RELIEVEv11n1_1.htm

Canales, R. y Marqués, P. (2007). Factores de buenas prácticas educativas con apoyo de las TIC. Análisis de su presencia en tres centros educativos. Educar, 39, 115-133. Recuperado de http://www.raco. cat/index.php/Educar/article/viewArticle/76748/0

Claro, M. (2010). La incorporación de tecnologías digitales en educación. Modelos de identificación de buenas prácticas. Documento de proyecto. Editorial CEPAL. Recuperado de http://archivo.cepal.org/ pdfs/2010/S2010481.pdf

Crompton, H. (2013). A historical overview of M-Leaning. Toward Learner - Centered Education. En Z.L. Berge y L.Y. Muilenburg (Eds.). Handbook of Mobile Learning. Londres: Routledge.

Del Barrio, A. y Ruiz, I. (2014). Los adolescentes y el uso de las redes sociales. International Journal of Developmental and Educational Psychology, 1(3), 571-576, ISSN: 0214-9877.

Fundación Omar Dengo [FOD]. (2006). Educación y tecnologías digitales. Cómo valorar su impacto social y sus contribuciones a la equidad. San José: Fundación Omar Dengo. Recuperado de: http://aplicaciones02.fod.ac.cr/idrc/intro.htm

Fundación Omar Dengo [FOD]. (2015a). Informe final del proceso de evaluación del proyecto Aprendizaje con Tecnologías Móviles en escuelas multigrado (Documento interno). Unidad de Evaluación, Fundación Omar Dengo. San José, Costa Rica.

Fundación Omar Dengo [FOD]. (2015b). Informe final de evaluación del proyecto Rem@, 20122014. (Documento interno). Unidad de Evaluación, Fundación Omar Dengo. San José, Costa Rica.

Fundación Omar Dengo [FOD]. (2015c). Desarrollo de competencias científicas en estudiantes de primaria: Movilab Primaria. Informe final de evaluación. (Documento interno). Unidad de Evaluación, Fundación Omar Dengo. San José, Costa Rica.

Fundación Omar Dengo [FOD]. (2016a). Tecnologías digitales y capacidades para construir el futuro: aportes del Programa Nacional de Informática Educativa MEP-FOD. FOD: San José, Costa Rica.

Fundación Omar Dengo [FOD]. (2016b). Informe final de evaluación: Unidades Móviles para el aprendizaje de las Matemáticas y el Español: MoviLab Secundaria. (Documento interno). Unidad de Evaluación, Fundación Omar Dengo. San José, Costa Rica.

Fundación Omar Dengo [FOD]. (2018). Diseño de estudio de la propuesta educativa con Tecnologías Móviles: Movilab Secundaria Diurno. (Documento interno). Unidad de Evaluación FOD: San José, Costa Rica.

Gómez, L. (2008). Los determinantes de la práctica educativa. Universidades, 38, 29-39. ISSN 0041-8935. Recuperado de https://www.redalyc.org/pdf/373/37303804.pdf

Hernández, R., Fernández, C. y Baptista, P. (2014). Metodología de la Investigación. Quinta Edición. McGraw-Hill. Interamericana Editores, S.A.

Instituto Nacional de Estadística y Censo [INEC]. (2014). Clasificación de Actividades Económicas de Costa Rica 2011. San José, Costa Rica: INEC.

Ministerio de Planificación Nacional y Política Económica [MIDEPLAN]. (2018). Índice de Desarrollo Social de Costa Rica 2017. San José, CR: MIDEPLAN

Pérez, R. (2011). Innovación educativa con una computadora por alumno: Factores facilitadores del Plan CEIBAL. En C. Guedez, V. Machado y L. Rodríguez (compiladores), La gestión, desafío crítico para la calidad y la equidad educativa (pp. 152-208). Montevideo: MEC. Dirección de Educación. 
Ping Lim, C., Aubé, M., Wagner de Huergo, E., Kalaš, I., Laval, E., Meyer, F., Rjazanova, J., Yong Tay, L. y Tokareva, N. (2014). ICT in Primary Education, Analytical survey. Volume 3: Collective Case Study of Promising Practices. UNESCO: Institute for Information Technologies in Education.

Said, E., Silveira, A., Valencia J., Iriarte, F., Justo, P., \& Ordoñez, M. (2015). Factores asociados al nivel de uso de las TIC como herramienta de enseñanza y aprendizaje en las instituciones educativas oficiales de Colombia y Brasil. Universidad del Norte. Recuperado de https://dialnet.unirioja.es/descarga/ libro/579342.pdf

\section{Agradecimientos:}

A Melania Brenes Monge, quien coordinó el diseño del estudio durante el año 2018 y a las investigadoras Vivian Rivera Ramírez y Angélica Zúñiga Baldi quienes estuvieron a cargo del proceso de recolección de los datos.

Para citar este documento:

Villalobos-García, M. y Núñez-Sosa, O. (2020). Prácticas pedagógicas apoyadas por tecnologías móviles: oportunidades para potenciar el aprendizaje de la población estudiantil. Revista Innovaciones Educativas. 22(32), 78-90. DOI: https://doi.org/10.22458/ie.v22i32.2806 